\title{
Systematic medication review and health-related outcome in elderly patients acutely admitted to an orthopaedic ward: a randomised controlled study
}

\author{
Marianne Lisby ${ }^{1,2^{*}}$, Dorthe Krogsgaard Bonnerup ${ }^{3}$, Birgitte Brock ${ }^{1,4}$, Pernille Axel Gregersen ${ }^{1}$, Jørn Jensen ${ }^{5}$, Jørgen Rungby ${ }^{4,6}$ \\ From Danish Society for Emergency Medicine: Research Symposium 2010 \\ Roskilde, Denmark. 20-21 May 2010
}

\section{Background}

Acute admission to hospitals, age and number of drugs are associated with an increased risk of adverse drug events due to morbidity, poly-pharmacy and inappropriate interactions. Thus, the objective of the present study was to investigate if systematic medication review and drug counseling on admission to hospitals would have a significant impact at clinically health-related outcomes in elderly patients admitted acutely to an orthopaedic ward.

\section{Methods}

A randomised controlled study comprising 108 patients aged 65 years or older admitted acutely to an orthopaedic ward in Denmark was conducted. Intervention: a clinical pharmacist collected information regarding the patient's medication and medical history in the medical record, the electronic drug order system, the registry of drug purchase and interview with the patient's. Subsequently, this was discussed with a clinical pharmacologist and an advisory note with drug recommendations were handed directly to the ward physicians. Control: the usual medication routine in the ward. The primary outcome measure was time to first unscheduled contact to a physician post-discharge limited to emergency department visit, readmission and general practitioner. Secondary outcomes were admission time, time to first re-admission, number of re-admissions, emergency department visits, ambulatory care visits, contact to general practitioner, physicians outside working hours, medical specialist and finally, quality of life at three-month follow up.

* Correspondence: marilisb@rm.dk

${ }^{1}$ Department of Clinical Pharmacology, Aarhus University Hospital, Denmark Full list of author information is available at the end of the article

\section{Results}

In the intervention group time to first unscheduled contact to a physician post-discharge was 16.3 days $(95 \% \mathrm{CI}$ : 10.5 $22.2)$ and in the control group 25.6 days (95\%CI:17.8-33.4), which was not a statistically significant difference. No overall differences between study groups were seen in the secondary outcome measures. The physicians complied with 41 of 222 drug recommendations. Subgroup analysis indicated that physicians were more prone to accept recommendations in the very old treated with numerous of drugs.

\section{Conclusion}

The findings do not lead to the recommendation of an extra medication review in a general population of elderly admitted acutely to an orthopaedic ward. Considering the physicians marked hesitation to comply with drug recommendations future studies should explore the impact of a differentiated medication review in acute admitted patients based on the individual patient's risk of adverse drug events.

\section{Author details \\ 'Department of Clinical Pharmacology, Aarhus University Hospital, Denmark. ${ }^{2}$ Center of Emergency Medicine Research, Aarhus University Hospital, Denmark. ${ }^{3}$ The Pharmacy Department, Aarhus University Hospital, Denmark. ${ }^{4}$ Department of Pharmacology, Aarhus University, Denmark. ${ }^{5}$ The Orthopeadic Department, Randers and Grenaa Regional Hospital, Denmark. ${ }^{6}$ Department of Medical Endocrinology, Aarhus University Hospital, Aarhus Sygehus, Denmark.}

Published: 17 September 2010

doi:10.1186/1757-7241-18-S1-P31

Cite this article as: Lisby et al:: Systematic medication review and healthrelated outcome in elderly patients acutely admitted to an orthopaedic ward: a randomised controlled study. Scandinavian Journal of Trauma, Resuscitation and Emergency Medicine 2010 18(Suppl 1):P31. 